\title{
Weighted Energy Efficient Cluster Based Routing for Wireless Sensor Networks
}

\author{
Soumyabrata Saha ${ }^{1}$ and Rituparna $\mathrm{Chaki}^{2}$ \\ ${ }^{1}$ Dept. of Computer Sc. \& Engg. \\ University of Calcutta, West Bengal, India \\ som. brata@gmail.com \\ ${ }^{2}$ Dept. of Computer Sc. \& Engg. \\ West Bengal University of Technology, West Bengal, India \\ rituchaki@gmail.com
}

\begin{abstract}
Wireless sensor network comprises of numerous tiny sensor nodes to form an ad hoc distributed sensing and data propagation network to collect the context information on the physical environment. Wireless sensor networks are used for remotely monitoring tasks and effective for data gathering in a variety of environments. Minimizing energy dissipation and maximizing network lifetime are one of the central concerns to design applications and protocols for wireless sensor networks. Cluster based architectures are one of the most practical solutions in order to cope with the requirements of large scale of sensor networks. Clustering results in a reduced number of messages that propagate through the network in order to accomplish a sensing task. In this paper, we have presented a brief review of the state of the art scenario of various routing protocols and propose a weighted energy efficient cluster based routing for wireless sensor networks. Conducted simulation experiments on different scenarios shown its ability to obtain effective \& efficient communications among different clusters and achieves scalability in large scale of wireless sensor networks.
\end{abstract}

Keywords: Wireless Sensor Networks, Weighted, Energy, Cluster, Routing.

\section{Introduction}

Wireless sensor networks are formed by densely and usually randomly deployed large number of sensor nodes either inside or very close to the phenomenon that is being monitored. The potential applications of sensor networks are highly varied, such as natural phenomena, environmental changes, controlling security, estimating traffic flows, monitoring military application, and tracking friendly forces in the battlefields.

Wireless sensor networks consist of sinks and sensors. Sinks play a role of collecting data which is transmitted by sensors. Sensor nodes sense the desirable physical phenomenon and locally do the data aggregation to avoid communication of redundant data. Using routing protocol sensor nodes determine the path for sending data to sink. A sensor node is comprised of four basic components: sensing unit, processing unit, radio unit and power unit. The sensing unit is used to measures a 
certain physical condition. Processing unit is responsible for collecting and processing signals. The radio unit transfers signals from the sensor to the user through the gateway. All previous units are supported by the power unit to supply the required energy in order to perform the mentioned tasks.

Clustering in wireless sensor networks provide scalability and robustness for the network. It allows spatial reuse of the bandwidth, simpler routing decisions, and results in decreased energy dissipation of the whole system by minimizing the number of nodes that take part in long distance communication. A cluster is a group of linked nodes working together closely for same purposes and belongs to same topological structure. A cluster head is responsible of resource allocation to all nodes belonging to its cluster and directly associated to its neighbor clusters for performing various task of intra and inter cluster communication. Sensor network used to be designed as clustering structure to minimize transmission costs and energy usage to prolong the network lifetime.

In this paper we propose a weighted energy efficient cluster based routing for wireless sensor networks. This proposed routing scheme comprises of five different steps. At first cluster head selection mechanism has been executed. The main motive of dynamic cluster head rotation mechanism is to evenly distribute the energy load among all the sensor nodes so that there are no overly utilized sensor nodes that will run out of energy before the others. Cost calculation technique has been introduced in second stage. Depends upon the dynamic cluster head selection and cost estimation technique, cluster formation mechanism has been presented at third step. Communication procedure has been described at step four. In step five route maintenance mechanisms take an important role to maintain the routes to deliver the messages from sender to receiver.

The rest of the paper is organized as follows. A comprehensive survey of related works of different routing techniques in Wireless Sensor Networks is presented in Section 2. In Section 3, we have design and describe a new routing algorithm, which incurs the transitions among these five different phases and improves energy efficiency to prolong the whole network lifetime. Energy Estimation mechanism is described in section 4. Intensive result analysis is presented in section 5. Finally, we conclude our paper with final remarks in Section 6.

\section{Related Works}

In recent years, cluster based architectures are one of the most suitable solutions to cope with the requirements of large scale wireless sensor networks. The clustering protocols have been extensively studied in this literature, which mainly differ in the selection of cluster heads, reformation strategies, hopping limits and routing scheme. In this section we take a brief look at some of the common clustering algorithms applicable for wireless sensor networks.

In HCBQRP [1] the main objective was to design a cluster based routing algorithm for sensor networks to find route from source to destination. HCBQRP [1] was designed based on the query-driven approach and provides the real time communication between sensor nodes.

Maximum Degree and Negotiation Strategy Based Clustering Algorithm for wireless sensor networks [2] was proposed by Qiang Wang et.al. MXAD-N [2] is 
divided into two phases; one is communication and computation of node degree and other is cluster head election and cluster formation. MAXD-N [2] selects candidate based maximum degree and determines cluster head according to the negotiation strategy.

Babar Nazir et.al. proposed Mobile Sink Based Routing Protocol [3] for prolonging network lifetime in clustered wireless sensor network. In MSRP [3] mobile sink moves within cluster area to collect sensed data from cluster heads and maintains information about the residual energy of the cluster head.

In [4] Tian Ying et.al. presented Energy Efficient Chain Cluster Routing protocol for wireless sensor networks. ECRM [4] protocol divided into backbone setup phase, cluster formation phase and steady communication phase. ECRM [4] includes efficient cluster head selection mechanism to prolong the network lifetime and improve the energy efficiency.

Asif U. Khattak et.al. proposed a Two Tier Cluster Based Routing Protocol [5] for wireless sensor networks. TTCRP [5] configures the nodes in the form of clusters at two levels. TTCRP [5] introduced a power control algorithm to allow the isolated sensor nodes as well as cluster heads to dynamically change their transmission power and provides network robustness.

LU Li fang et.al proposed Weight Based Clustering Routing Protocol for wireless sensor networks [6]. In WCR [6] cluster head selection algorithm is designed for periodically select cluster heads based on the node position and residual energy. WCR [6] is used to minimize the energy dissipation of intra cluster and inter cluster data transmission.

In [7] Awwad et.al. presented adaptive time division multiple access scheduling and round free cluster head protocol called Cluster Based Routing Protocol for Mobile nodes in Wireless Sensor Network [7]. CBR Mobile-WSN [7] is energy aware scheme and used to handle packet loss and reduces the energy consumption.

An Energy Level Based Routing Algorithm of Multi sink Sensor Networks [8] was proposed by Zhongbo et.al. In ECR [8] two different hierarchy structure of the network topology are presented. The operation of ECR [8] can be divided into three steps, cluster-formation phase, cluster head selection phase and steady state phase.

Lu Cheng et.al. proposed an Energy Efficient Weight Clustering Algorithm [9] to reduce energy consumption by perfecting cluster formation procedure. In EWC [9] residual cluster energy, location and node degree and coefficients are takes an important role in cluster head selection stage and nodes' having minimal combined weight becomes cluster head.

In [10] A. Martirosyan et al. presented an Energy Efficient Inter Cluster Communication based routing protocol for WSNs. ICE [10] uses acknowledgement based approach to faulty paths discovery and provides QoS by finding a path with the least cost for high priority event notification messages.

An Adaptive Decentralized Re-clustering Protocol for wireless sensor networks [11] was proposed by Sethares et.al. ADRP [11] incurs high overhead in forming clusters due to the information exchanged between the nodes and the sink

Boukercheet. al. proposed Clustering PEQ [12], configures the dissemination tree using the PEQ's [12] mechanism and an additional field contains the percentage of nodes that can become cluster head. CPEQ [12] employs an energy aware cluster head selection mechanism in which the sensor nodes with more residual energy are selected as cluster head and increases the network lifetime. 
Kyung Tae Kim et.al. presented an Energy Driven Adaptive Clustering Hierarchy for WSNs [13] to increase the lifetime of sensor networks. EDACH [13] protocol is based on partitioning of the network according to the relative distance to the base station for assigning different probability of cluster head.

In [14] Proxy Enabled Adaptive Clustering Hierarchy proposed by Kim et.al. A proxy node assumes as cluster head in place of weak cluster head during one round of communication. In PEACH [14], the clustering scheme avoids long range communication, data fusion and saves energy by compressing the data and rotation of cluster head allows to prolong the lifetime of every node.

An Energy Efficient Unequal Clustering [15] routing protocol was proposed by Hee Yong Youn et.al. EEUC [15] is a distributed competitive algorithm, where cluster heads are elected by locally and clusters are closer to the base station are expected to have smaller cluster sizes.

In [16], according to free space model, the propagation loss is modeled as inversely proportional to $\mathrm{d}^{\mathrm{i}}$ (where $\mathrm{i}=2$, if $\mathrm{d}<\mathrm{d}_{0}$ ). In the multi-path fading channel model [16], the propagation loss is modeled as inversely proportional to $d^{i}$ (where $i=4$, if $d \geq d_{0}$ ). At the time of transmitting message, the amount of energy dissipated by sensor node has been defined as; $\quad E P_{t x}(p, d)=E P_{\text {elec }} * p+\varepsilon_{f s} * p * d^{i}$ To receive this $\mathrm{p}$-bit message the energy dissipated; $E P_{r x}(p)=E P_{\text {elec }} * p$ Where $\mathrm{p}$ is the size of message, $\mathrm{d}$ is the distance between source and destination node, $E P_{\text {elec }}$ is the circuit energy cost for transmitting or receiving purposes, $\varepsilon_{\mathrm{fs}}$ is the amplifier parameter.

In [17], the first order radio model is used, which consists of three main models: the transmitter, the power amplifier, and the receiver. The amount of energy consumed by each sensor node while transmitting message, is defined as

$$
E P_{t x}(p, d)=E P_{\text {elec }} * p+E P_{a m p} * p * d^{2} \text {, where } E P_{\text {amp }} \text { is the amplifier coefficient. }
$$

Heinzelman et.al. proposed Low Energy Adaptive Clustering Hierarchy [17] algorithm for sensor networks. Two layers architecture was introduced in LEACH [17]. One used for communication within the clusters and the other was between the cluster heads and sink. Due to the random selection, there exists the probability of unbalanced cluster head selection and selected cluster head may be present in one part of the network, making other portion of the network unreachable.

Through the extensive literature review it is observed that the selection of cluster heads can greatly affect the performance of the whole network and well selected cluster heads not only decrease the energy consumption but also prolong the network lifetime. Energy efficiency is unanimously considered as core design issue and in order to improve the deficiencies of aforementioned schemes, the challenge is to develop a routing protocol that can meet these conflicting requirements while minimizing compromise. In the next section we are going to propose a new weighted energy efficient cluster based routing protocol for wireless sensor networks.

\section{Proposed New Routing Protocol}

The previous section leads to the observation of different cluster based routing protocol for wireless sensor networks. Clustering involves in the cluster formation techniques where low energy nodes are assigned the task of sensing. The main aim of 
cluster based routing algorithm is to efficiently maintain the energy consumption of sensor nodes by involving them in multi hop communication.

The assumptions made for this protocol are as follows: all sensor nodes in the network are immobile, homogeneous and are equipped with power control capabilities; sensor nodes are generally energy constrained and they are capable to operate in an active mode or a low power sleeping mode and have enough processing power to support the different protocols and data processing tasks; each sensor node has a unique identifier and uniformly deployed over the target area to continuously monitor the environment; every sensor node can directly communicate with its immediate neighbor; the transmission range of each node is same on one condition.

Wireless sensor network is usually data centric. Collected data are periodically transmitted from the sensor node to the remote sink node. It is assumed that the sensed information is highly correlated, thus the cluster head can always aggregate the data gathered from its members into a single length fixed packet. The correlation degree of sensed data from different clusters is relatively low.

In the proposed weighted cluster based routing algorithm protocol, WSN perceived as a network partitioned into different clusters and we present a new robust approach of routing. The proposed weighted cluster based routing scheme is divided in some subsections. In section 3.1 cluster head selection procedures has been presented. Section 3.2 is used for cost calculation technique. In section 3.3 we have described cluster formation mechanism. Section 3.4 presented communication procedure. Route maintenance technique has been presented in section 3.5.

\subsection{Cluster Head Selection Procedure}

In our proposed scheme we introduce a weight based cluster head selection procedure. The main objective of choosing cluster head that guarantees both the intra-cluster and inter-cluster data transmission with energy efficient manner. Many energy consuming activity have to perform by the cluster heads, such as, data collections from member nodes and forwarding processed data to other neighbors or to the sink node. To select the cluster head, we have considered some parameters like; number of neighbor nodes, distance between cluster heads and sink node, degree difference of node; residual energy of sensor node is another important parameter for cluster head selection process. Every sensor node takes part in cluster head selection procedure. A node is selected as a cluster head and its energy level is periodically monitored. Once the energy level goes below the threshold value, it is released from its' special responsibilities.

We assume that $\mathrm{N}$ number of sensor nodes are scattered randomly over the area of interest and left unattended to continuously sense and report events. We denote the $i_{t h}$ sensor by $s_{i}$ and the corresponding sensor node set $S=\left\{s_{1}, s_{2}, \ldots ., s_{N}\right\}$, where $|S|=N$, where $\mathrm{N}$ is the total number of sensor nodes in the network.

Definition 1: At time instance $T_{n}$, to send a p-bit message from transmitter to receiver at the distance $d_{i}$ and to receive the p-bit message at same distance $d_{i}$, the total energy dissipated from node $s_{i}$ is $E_{\text {req }}$

$$
E_{\text {req }}=\left\{\left(E P_{\text {elec }} * p+\varepsilon_{f s} * p * d^{2}\right)+\left(E P_{\text {elec }} * p\right)\right\}
$$


And the remaining energy for node $\mathrm{s}_{\mathrm{i}}$ is $\mathrm{E}_{\mathrm{r}}$

$$
E_{r}=\left\{\left(E_{\text {init }}\right)-\left(E_{\text {req }}\right)\right\}
$$

After calculating total dissipated energy and remaining energy of node $s_{\mathrm{i}}$ for time instance $\mathrm{T}_{\mathrm{n}}$, store all these different estimated energy in the ND_ENGY_TBL $\left\{\boldsymbol{S}_{\boldsymbol{i}}\right.$, $\left.\boldsymbol{E}_{\text {init }}, \boldsymbol{E}_{\text {req }}, \boldsymbol{E}_{\boldsymbol{r}}, \boldsymbol{T}_{\boldsymbol{n}}\right\}$ table.

\section{Algorithm 1. Algorithm for Cluster Head Selection}

Step1: Find the neighbors of each node $\mathrm{s}_{\mathrm{i}}$

$$
\mathrm{N}^{\mathrm{N}} \hat{\mathrm{N}}_{s_{i}}[]=\sum \dot{s}_{i} € \hat{\mathrm{N}}, s_{i} \neq \dot{s}_{i}\left\{\operatorname{dist}\left(s_{i}, \dot{s}_{i}\right) \leq t_{r}\right\}
$$

Step2: $\quad$ Compute the distance $d s_{i}$ for each node $\mathrm{s}_{\mathrm{i}}$ as:

$$
d_{s_{i}}=\operatorname{dist}\left(s_{i}, d_{\text {snk }}\right)
$$

Step3: Compute the degree difference for every node $\mathrm{s}_{\mathrm{i}}$

$$
\Delta s_{i}=\sum \dot{s}_{i} € \hat{\mathrm{N}},\left[\left\{\mathrm{N}_{\mathrm{g}} \hat{N}_{s_{i}}-\dot{\varepsilon}\right\} \leq t_{r}\right]
$$

Step4: Read initial energy $E_{i n i t}$ and residual energy $E_{r}$ of node $s_{i}$ for each time instance $\mathrm{T}_{\mathrm{n}}$ from ND_ENGY_TBL and update this energy table.

Step5: Calculate the total weight of each node $\mathrm{s}_{\mathrm{i}}$ as:

$$
w_{s_{i}}=\left[w_{1} * \mathrm{~N}^{\prime} \hat{N}_{s_{i}}+w_{2} * d_{s_{i}}+w_{3} * \Delta s_{i}+w_{4} * E_{\text {req }}+w_{5} * T_{\text {tach }}\right]
$$

Step6: Store all value of $w_{s_{i}}$ in the $\mathrm{w}_{\mathrm{s}}[]$ and select minimum value of $w_{s_{i}}$ as $C h \_I d_{i}$ Step7: END.

By using the above technique, cluster head selection has been executed.

\subsection{Cost Calculation}

In this section we propose a method of calculating combined cost between various sensor nodes. After a brief survey of network environment, we are going to define some network parameters. These parameters reflect the cost of hopping to its neighbors, i.e., the cost of a link leading from the node to its neighbors. The parameters are described below:

$\operatorname{tp}_{\mathrm{i}} \quad$ The throughput of sensor network. This can be estimated by counting total received and send packet by node $i$ in a time interval.

$b_{\mathrm{i}} \quad$ The available bandwidth of NIC of node $i$.

$\mathrm{sb}_{\mathrm{i}} \quad$ Indicates the saturation of sending buffer at node $i$. If the length of buffer queue reaches $85 \%$ of its total size then $\mathrm{sb}_{\mathrm{i}}$ set to " 1 "; otherwise sb $b_{i}$ set to " 0 ".

$\mathrm{rb}_{\mathrm{i}} \quad$ Indicates the saturation of receiving buffer at node $\mathrm{i}$. If the length of buffer queue reaches $85 \%$ of its total size then $\mathrm{rb}_{i}$ set to " 1 "; otherwise rb $b_{i}$ set to " 0 ".

By using the above parameters, we can estimate communication cost between two nodes as follows:

$$
\operatorname{Cost}_{\text {comm }}=\left[\left\{\left({ }^{t p_{i}} / b w_{i}\right) * w_{s}\right\}+s b_{i}+r b_{i}\right]
$$


The total cost can be calculated using this following equation:

$$
\text { Cost }_{i, j}=\left[\left\{\text { Cost }_{\text {comm }}\right\}+\left\{\text { Cost }_{\text {energy }}\right\}\right]
$$

\subsection{Cluster Formation Mechanism}

After cluster head selection process is completed, each cluster head broadcast a CM_FRM_MSG $\left\{\boldsymbol{C h} \boldsymbol{I} \boldsymbol{d}, \boldsymbol{M s} \boldsymbol{g} \_\boldsymbol{I d}, \boldsymbol{C h} \_\boldsymbol{W g}, \boldsymbol{T T} \boldsymbol{L}\right\}$ message to its neighbor nodes.

If a node receives this type of message from more than one cluster head, then it chooses to join the membership of the cluster head causing minimum cost. Accordingly, the node sends a reply CM_RPLY_MSG $\left\{\boldsymbol{C h} \_\boldsymbol{I d}, \boldsymbol{C m} \_\boldsymbol{I d}, \boldsymbol{C h} \_\boldsymbol{W g}\right.$, Msg_Id, Cost $\left.t_{i, j}, \boldsymbol{T T L}\right\}$ message to the corresponding cluster head with minimum total cost.

If any other cluster head receives this CM_FRM_MSG message from the other cluster head, then it will truncate the message and don't send any reply or acknowledgement to the sender cluster head. If any cluster member of other cluster again receives this message, then it will follow the same procedure. The total process is described below.

Algorithm 2. Algorithm for Cluster Formation

Step 1: $\quad$ Ch_id ${ }_{i}$ broadcast a CM_FRM_MSG message for cluster formation.

For each node in the network:

Step 2: If the node is already another cluster head or member of another cluster

Then it discards the previous message.

Else

Step I: Calculates total cost between it and Ch_id

Step II: If total cost $<\mathrm{Th}_{\text {cost }}$

Then it sends a reply CM_RPLY_MSG message to $\mathrm{Ch}$ id $_{\mathrm{i}}$ and added to $\mathrm{Ch} \_$id $\mathrm{i}_{-} \operatorname{Mem}[\mathrm{]}$.

Else

Then it discards the previous message.

Step 3: $\quad$ END.

By using the above procedure any non-member nodes can join to the cluster head.

\subsection{Communication Procedure}

In this section we have discussed the communication procedure between sensor nodes. Every cluster head maintains the NGH_CLSRHD_TBL $\left\{\boldsymbol{C h} \_\boldsymbol{I d}, \boldsymbol{C h} \_\boldsymbol{W g}\right.$, Ch_Comm_Cost, $\boldsymbol{T T L}\}$ table and also maintains their cluster members' information in CM_INF_TBL $\left\{\boldsymbol{C} \boldsymbol{m} \_\boldsymbol{I d}, \boldsymbol{M s g} \boldsymbol{I} \boldsymbol{I}, \boldsymbol{C} \boldsymbol{C} \_\boldsymbol{W g}, \boldsymbol{C o s t} \boldsymbol{c o m m}_{\text {com }}\right\}$ table.

When any sensor node requires some information, it initiates communication. At first it communicates with its corresponding cluster head and cluster head searches in its CM_INF_TBL table. If required information is not available then it searches in NGH_CLSRHD_TBL table. The cluster head with lowest communication cost is selected as next hop. The algorithm is described below: 
Algorithm 3. Algorithm for Communication Procedure

Step 1: $\quad$ Node $\mathrm{N}$ send a request message to corresponding $\mathrm{Ch}_{\mathrm{i}}$.

Step 2: $\quad \mathrm{Ch}_{\mathrm{i}}$ searches in its CM_INF_TBL.

Step 3: If required information is available

Step I: Then it appends corresponding node's Cm_Id in RT_INFO table.

Step II: That node sends message to sink and updates its energy level.

Step III: Communication end.

Else

Step I: Searches in NGH_CLSRHD_TBL

Step II: The $\mathrm{Ch}_{\mathrm{i}}$ with lowest communication cost selected as next hop.

Step 4: END.

Step III: $\quad$ Go to step 2.

After successful execution of the above algorithm we find the node with required information. This node becomes source node and sends a message including the required information through selected path from RT_INFO $\left\{\boldsymbol{S n d} \_\boldsymbol{N d} \_\boldsymbol{I d}, \boldsymbol{N} \boldsymbol{x t} \_\boldsymbol{H}\right.$, Dst_Id\} table.

\subsection{Route Maintenance Techniques}

If in a particular route, a sensor node is damaged for a certain time period or link is broken, the route will be damaged. To handle this kind of situation we have incorporated route maintenance mechanism to overcome this problem.

After receiving any information from any sensor node, the receiving node has send ACK_MSG $\left\{\boldsymbol{S n d} \_\boldsymbol{N} d \_I d, \boldsymbol{R e c v r} \_N d \_I d, M s g \_I d, \boldsymbol{A c k} \_I d, T \boldsymbol{T} \_S t m p\right\}$ to the sender node within a certain time period. These ACK_MSG message based repair mechanisms consist of two parts. One is failure detection and other is selection of alternative node.

Depends on the reception time of ACK_MSG sensor nodes can detect if its neighbor nodes are functioning properly or not. If ACK_MSG not received within a specified time, the sensor node initiates the communication procedure to find out the alternative route.

By using the above discussed algorithm any sensor node can communicate with any another nodes through the cluster head within that network.

Algorithm 4. Algorithm for Route Maintenance

Step1: If ACK_MSG is not received within specified time interval

Then execute communication procedure for finding alternative route

Else terminate the process.

Step2: END. 
Table 1. Data Dictionary

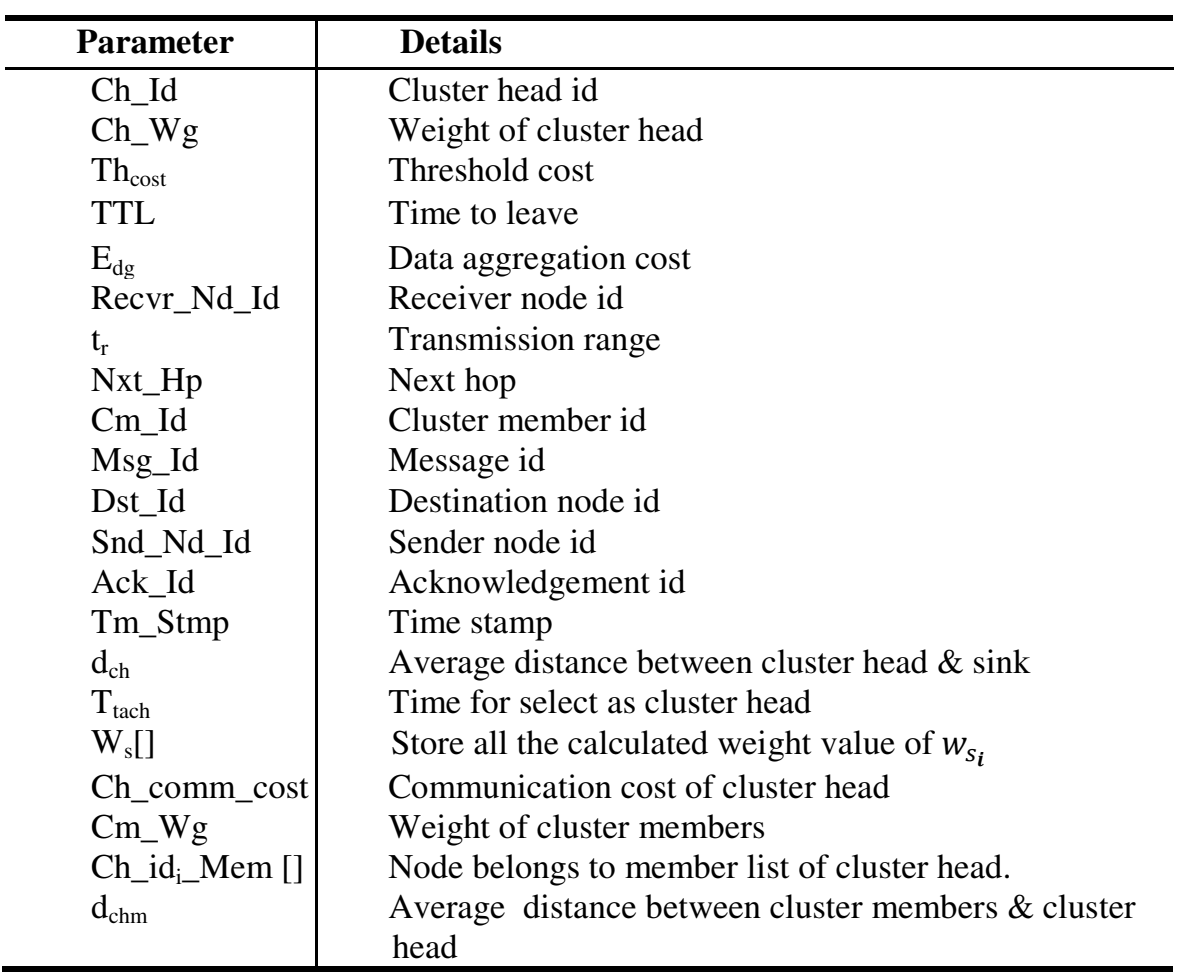

\section{$4 \quad$ Energy Estimation}

Definition 2: In the proposed cluster based network, assuming there are n numbers of cluster members and each member having the capacity of sending at most p-bit message. The total number of the received message at the cluster head is $\mathrm{m}$;

$$
\begin{gathered}
m=n * p \\
E_{c m}=\left[E P_{\text {elec }} * p+\varepsilon_{f s} * p * d_{c h m^{2}}\right]
\end{gathered}
$$

Definition 3: Eqn (x) represents average energy consumption per cluster member, assuming there are $n$ numbers of cluster members are present within the cluster.

$$
E_{a m}=\left\{1 / n \sum_{i=1}^{n} E_{c m}(i)\right\}
$$

Definition 4: The energy consumed by a cluster head is obtained by Eqn (xi). $\mathrm{E}_{\mathrm{ch}}$ is the energy consumption as the cluster head transmits the aggregated data to the sink node. 


$$
E_{c h}=E P_{\text {elec }} * m+\varepsilon_{f s} * m * d_{c h^{2}}
$$

Definition 5: Eqn (xii) represents average energy consumption per cluster head, assuming there are $c$ number of clusters are present in the network. Since $E_{\text {ach }}$ change over time, the threshold is calculated in every data collection and transmission phase.

$$
E_{a c h}=1 / c \quad \sum_{i=1}^{c} E_{c h}(i)
$$

Definition 6: For our proposed weighted energy efficient cluster based routing scheme, the energy dissipated during a round is given by Eqn (xiii).

$$
\begin{gathered}
E_{\text {chm }}=\left[\left(E P_{\text {elec }} * m+\varepsilon_{f s} * m * d_{\text {chm }}{ }^{2}\right)+\left(\sum _ { i = 1 } ^ { n } \left\{E P_{\text {elec }} * p+\varepsilon_{f s} * p *\right.\right.\right. \\
\left.\left.\left.d_{c h m^{2}}\right\}\right)+\left(E_{d g} * n * p\right)+\left(E P_{\text {elec }} * c * m\right)\right]
\end{gathered}
$$

Where $\mathrm{c}$ is the number of clusters present in the network and $\mathrm{m}$ is the number of transmitted messages.

Definition 7: Assuming that the sensor nodes are uniformly distributed, it can be shown that; where $\mathrm{q}^{2}$ is the area of the monitoring filed.

$$
\begin{gathered}
d_{c h m^{2}}=\int_{0}^{x_{\max }} \quad \int_{0}^{y_{\max }}\left(x^{2}+y^{2}\right) * \rho(x, y) d x d y \\
d_{c h m^{2}}=\left\{\left(q^{2} / 2 * \Pi * r\right) * d\right\}
\end{gathered}
$$

Definition 8: The total energy dissipated in the network is equal to:

$$
\begin{aligned}
E_{\text {tot }}= & {\left[\left(\sum_{i=1}^{n}\left\{E P_{\text {elec }} * p+\varepsilon_{f s} * p * d_{\text {chm }}{ }^{2}\right\}\right)+\sum_{i=1}^{c} E_{\text {chm }}(i)+\left(E_{d g} * m\right)+\right.} \\
& \left.+\left(E P_{\text {elec }} * c * m\right)\right]
\end{aligned}
$$

The total consumed energy of the sensor network is depends up on the construction of the cluster, if the clusters are not constructed in an optimal way, the total consumed energy of the sensor network per round is increase.

\section{$5 \quad$ Result Analysis}

The simulation model consists of a network model that has a number of wireless nodes, which represents the entire network to be simulated. The network simulator, NS-2 is used to evaluate the performance of the proposed protocol WECRP and present the following metrics for comparing the performance of WECRP with the AODV.

End2End delay is the average difference between the time the first data bit is originated by an application and the time this data bit is received at this destination. Throughput is defined as the total number of message sent or received per time unit. In this experiment, throughput is measured in terms of bits delivered per time unit. End2End of the data packet is one of the performances metric in our proposed scheme. The results for this metric are shown in fig 1. 
Table 2. Simulation Environment Parameters

\begin{tabular}{l|l}
\hline \multicolumn{1}{c|}{ Parameter } & \multicolumn{1}{|c}{ Value } \\
\hline Channel & Wireless Channel \\
Propagation & Two Ray Ground \\
Antenna & Omni Antenna \\
Phy & Wireless Phy \\
Mac & $802 \_11$ \\
Number of nodes & 10 \\
WSNs field & $(0,0) \sim(500,500) \mathrm{m}$ \\
Initial energy & $0.5 \mathrm{~J}$ \\
Data package size & 2100 bits \\
\hline
\end{tabular}

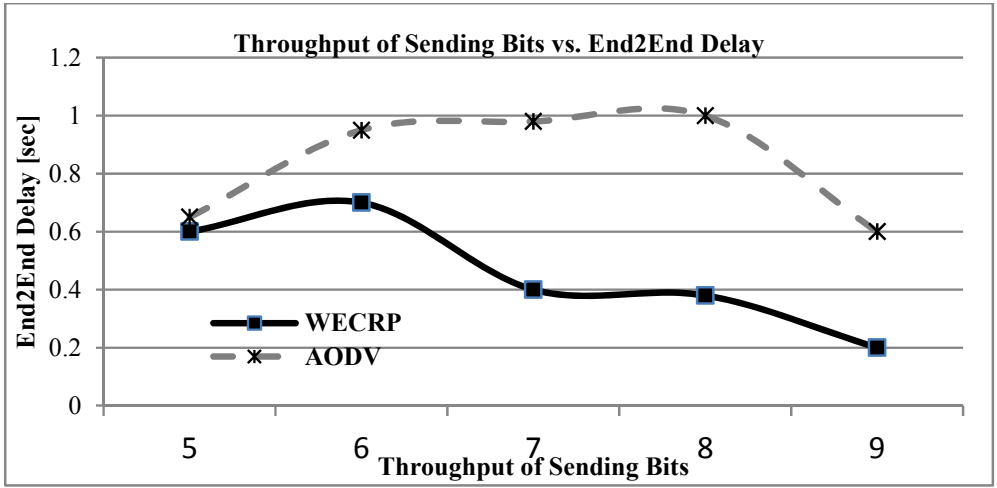

Fig. 1. Throughput of Sending Bits vs. End2End Delay

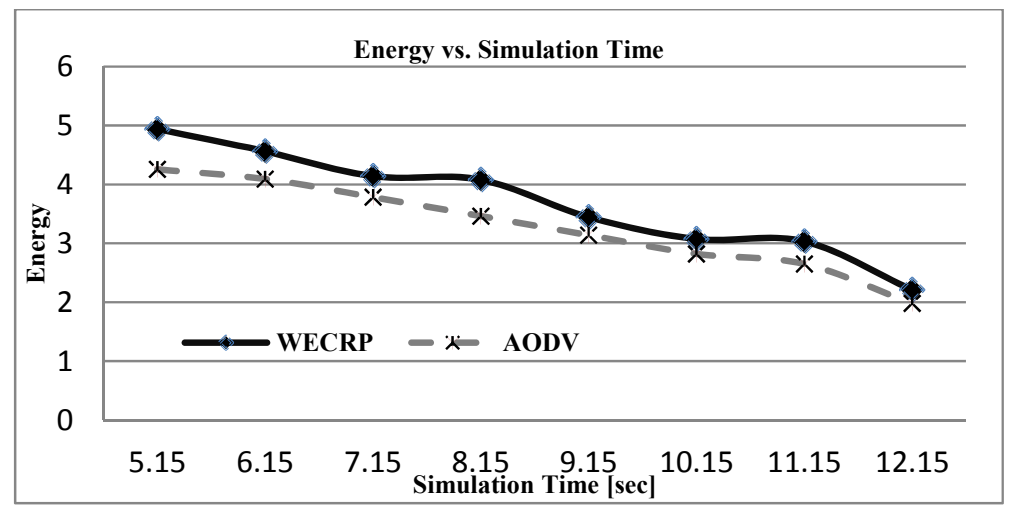

Fig. 2. Energy vs. Simulation Time 
From this graph we have observed that in all situations the delay of WECRP is lesser than AODV. Though our proposed protocol induces a lower routing load than AODV, as a result data transmissions are less delayed. Thus our approach with lower End2End delay offers better QoS.

In fig 2, we have depicted a relation between energy vs. simulation time. It has been observed that when more number of packet or data bit is transferred, corresponding energy are less dissipated compared to AODV.

\section{Conclusions}

In this paper we have summarized the generic characteristics of some well-known cluster based routing protocols for WSNs and present a new routing scheme, weighted energy efficient cluster based routing for Wireless Sensor Networks to achieve realtime communication and high energy efficiency. As the cluster head of each cluster acts as a local coordinator for its cluster, performing inter-cluster routing, data forwarding and has to undertake heavier tasks so that it might be the key point of the network. In our proposed routing scheme only cluster head can take part to share information with their neighboring cluster heads. In WECRP we have considered weight factor of every sensor nodes to select the cluster head. According to WECRP nodes having least weight factor will select as a cluster head. Using cost estimation technique each cluster head selects its neighbor cluster members. Within the same cluster or different clusters, communication procedure has been carried out and route maintenance takes an important role in this routing algorithm.

The routing of messages using the proposed WECRP is achieved in an efficiency manner as observed in the performance graph. The parameters used for performance analysis are energy efficiency, transmission delay and throughput. A comparative analysis of the proposed WECRP against AODV has been carried out. It has been observed that the proposed method shows better performance in each cases and achieved energy efficiency, scalability, information sharing and route maintenance mechanism.

\section{References}

1. Saha, S.B., Chaki, R.: HCBQRP: Hierarchical Cluster Based Query-Driven Routing Protocol for Wireless Sensor Networks. In: SPRINGER International Conference on Information Systems Design and Intelligent Applications, INDIA (2012)

2. Wang, Q., Wang, C., Wang, Y.: A Maximum Degree and Negotiation Strategy Based Clustering Algorithm for Wireless Sensor Networks. In: IEEE Instrumentation and Measurement Technology Conferences, I2MTC (2011)

3. Nazir, B., Hasbullah, H.: Mobile Sink based Routing Protocol for Prolonging Network Lifetime in Clustered Wireless Sensor Network. In: IEEE International Conference on Computer Applications and Industrial Electronics, ICCAIE (2010)

4. Ying, T., Yang, O.: A Novel Chain-Cluster Based Routing Protocol for Mobile Wireless Sensor Networks. In: 6th IEEE International Conference on Wireless Communications Networking and Mobile Computing, WiCOM (2010) 
5. Khattak, A.U., Shah, G.A., Ahsan, M.: Two-Tier Cluster Based Routing Protocol For Wireless Sensor Networks. In: IEEE/IFIP 8th International Conference on Embedded and Ubiquitous Computing, EUC (2010)

6. Lu, L.F., Lim, C.-C.: Weight-based Clustering Routing Protocol for Wireless Sensor Networks. In: IEEE International Symposium on IT in Medicine \& Education, ITIME (2009)

7. Awwad, S.A.B., Ng, C.K., Noordin, N.K., Rasid, M.F.A.: Cluster Based Routing Protocol for Mobile Nodes in Wireless Sensor Network. In: IEEE International Symposium on Collaborative Technologies and Systems, CTS (2009)

8. Wu, Z., Zhang, C., Chen, H.: Energy level based Routing Algorithm of Multi sink Sensor Networks. In: IEEE International Conference on Networking, Sensing and Control, ICNSC (2008)

9. Cheng, L., Qian, D., Wu, W.: An Energy Efficient Weight-clustering Algorithm in Wireless Sensor Networks. In: IEEE Japan-China Joint Workshop on Frontier of Computer Science and Technology (2008)

10. Boukerche, A., Martirosyan, A.: An Energy Aware and Fault Tolerant Inter cluster Communication based Protocol for Wireless Sensor Networks. In: Globecom, Washington, D.C. (2007)

11. Wen, C.Y., Sethares, W.A.: Adaptive Decentralized Re-Clustering for Wireless Sensor Networks. In: IEEE International Conference on Systems, Man and Cybernetics, SMC (2006)

12. Boukerche, A., Pazzi, R.W., Araujo, R.B.: Fault-tolerant wireless sensor network routing protocols for the supervision of context-aware physical environments. Journal of Parallel and Distributed Computing 66(4), 586-599 (2006)

13. Kim, K.-T., Youn, H.Y.: Energy-Driven Adaptive Clustering Hierarchy (EDACH) for Wireless Sensor Networks. In: Enokido, T., Yan, L., Xiao, B., Kim, D.Y., Dai, Y.-S., Yang, L.T. (eds.) EUC Workshops 2005. LNCS, vol. 3823, pp. 1098-1107. Springer, Heidelberg (2005)

14. Kim, K.T., Youn, H.Y.: PEACH: Proxy-Enable Adaptive Clustering Hierarchy for Wireless Sensor Networks. In: Proceedings of ICWN 2005, pp. 52-56 (2005)

15. Youn, H.Y., Kim, K.T.: An Energy Efficient Unequal Clustering Mechanism for Wireless Sensor Networks. In: IEEE International Conference on Mobile Ad hoc and Sensor Systems Conference (2005)

16. Heinzelman, W.R., Chandrakasan, A., Balakrishnan, H.: An Application-Specific Protocol Architecture for Wireless Micro sensor Networks. Wireless Communications 1(4), 660-670 (2002)

17. Heinzelman, W.R., Chandrakasan, A., Balakrishnan, H.: Energy-Efficient Communication Protocol for Wireless Micro sensor Networks. In: Proc. 34th Hawaii Int'. Conf. Sys. Sci. (2000) 